\title{
BMJ Open Acceptability and implementation challenges of smartphone-based training of community health nurses for visual inspection with acetic acid in Ghana: mHealth and cervical cancer screening
}

Ramin Asgary, ${ }^{1,2}$ Helen Cole, ${ }^{3}$ Philip Adongo, ${ }^{4}$ Ada Nwameme, ${ }^{4}$ Ernest Maya, ${ }^{4,5}$ Amanda Adu-Amankwah, ${ }^{5}$ Hannah Barnett, ${ }^{1}$ Richard Adanu ${ }^{6,7}$

To cite: Asgary R, Cole $\mathrm{H}$, Adongo P, et al. Acceptability and implementation challenges of smartphonebased training of community health nurses for visual inspection with acetic acid in Ghana: mHealth and cervical cancer screening. BMJ Open 2019;9:e030528. doi:10.1136/ bmjopen-2019-030528

- Prepublication history and additional material for this paper are available online. To view these files, please visit the journal online (http://dx.doi.org/ bmjopen-2019-030528).

Received 18 March 2019 Revised 18 June 2019 Accepted 20 June 2019

Check for updates

(C) Author(s) (or their employer(s)) 2019. Re-use permitted under CC BY-NC. No commercial re-use. See rights and permissions. Published by BMJ.

For numbered affiliations see end of article.

Correspondence to

Dr Ramin Asgary;

ga263@columbia.edu

\section{ABSTRACT}

Objective To explore acceptability and feasibility of smartphone-based training of low-level to mid-level health professionals in cervical cancer screening using visual inspection with acetic acid (VIA)/cervicography.

Design In 2015, we applied a qualitative descriptive approach and conducted semi-structured interviews and focus groups to assess the perceptions and experiences of community health nurses (CHNs) $(n=15)$ who performed smartphone-based VIA, patients undergoing VIA/ cryotherapy $(n=21)$ and nurse supervisor and the expert reviewer $(n=2)$.

Setting Community health centres (CHCs) in Accra, Ghana.

Results The 3-month smartphone-based training and mentorship was perceived as an important and essential complementary process to further develop diagnostic and management competencies. Cervical imaging provided peer-to-peer learning opportunities, and helped better communicate the procedure to and gain trust of patients, provide targeted education, improve adherence and implement quality control. None of the patients had prior screening; they overwhelmingly accepted smartphone-based VIA, expressing no significant privacy issues. Neither group cited significant barriers to performing or receiving VIA at CHCs, the incorporation of smartphone imaging and mentorship via text messaging. CHNs were able to leverage their existing community relationships to address a lack of knowledge and misperceptions. Patients largely expressed decisionmaking autonomy regarding screening. Negative views and stigma were present but not significantly limiting, and the majority felt that screening strategies were acceptable and effective.

Conclusions Our findings suggest the overall acceptability of this approach from the perspectives of all stakeholders with important promises for smartphonebased VIA implementation. Larger-scale health services research could further provide important lessons for addressing this burden in low-income and middle-income countries.

\section{Strengths and limitations of this study}

- Using a qualitative descriptive approach, this is the first paper that describes perceptions and experiences of nurses and patients in regards to smartphone-based visual inspection with acetic acid (VIA) for cervical cancer screening.

- Our findings provide important insight into the overall acceptability and feasibility of this approach from the perspectives of all stakeholders.

- The smartphone-based training of VIA has important promises for VIA implementation and sustainability of national programmes.

- This qualitative study by its nature only generates hypotheses, which need to be further tested quantitatively for validity and generalisability.

- Larger-scale health services research would further provide important lessons for addressing this burden in low-income and middle-income countries.

\section{INTRODUCTION}

Worldwide, cervical cancer kills around a quarter of a million women annually, with the majority in low-income and middle-income countries (LMICs). ${ }^{1-3}$ In sub-Saharan Africa (SSA), lack of access to proper screening and treatment of precancerous lesions contributes to high cervical cancer mortality. ${ }^{4-8}$ Visual inspection of the cervix under acetic acid (VIA) is an effective screening method for cervical cancer, ${ }^{9-20}$ but is not readily accessible in most SSA countries. ${ }^{10} 1920$ Periodic cervical cancer screening using VIA conducted every 24 months by trained primary health workers has resulted in a $31 \%$ reduction in cervical cancer mortality over 12 years. $^{21}$ Ghana's National Screening Programme recommends VIA screening plus cryotherapy for women 
aged 25-45 22 ; however, it has not been widely available due to an inability to integrate VIA into the primary healthcare system. ${ }^{5023}$ In Ghana, the Community-based Health Planning and Services (CHPS) programme has significantly improved access to primary care through community health nurses (CHNs), who provide services at the district level in community health centres $(\mathrm{CHCs}) .{ }^{24}{ }^{25}$

A shortage of healthcare providers and comprehensive training of low-level to mid-level healthcare workers in VIA contribute to the lack of VIA accessibility in SSA. ${ }^{6} 719$ 26-29 Additionally, maintaining competencies and accuracy of VIA are major challenges as the standard short-term onsite VIA trainings may not guarantee skills retention. ${ }^{19} 2829$ The addition of cervicography to VIA has improved accuracy and diagnostic abilities, ${ }^{30-35}$ but its logistical barriers and cost have impeded large-scale availability for practitioners. The use of smartphones with digital cameras has presented the opportunity to use smartphones for cervicography to enhance training of low- to mid-level health professionals, facilitate storage of patient's screening records, transfer images for expert opinion and re-train via text messaging. ${ }^{36-38}$ However, acceptability and feasibility of smartphone-based VIA have not been systematically evaluated and have implications for large-scale implementation. In a related 2016 pilot study, the authors have demonstrated the impact of smartphone-based imaging and mentorship on improving and maintaining VIA diagnostic skills among CHWs. ${ }^{36} \mathrm{In}$ this study, we aimed to evaluate the perceptions and experiences of both CHNs who performed smartphone-based VIA/cervicographyand women undergoing VIA/cryotherapy in CHCs in Accra, Ghana.

\section{METHODS}

\section{Setting and study participants}

The catchment area for this study included 15 CHPS demarcated zones in the Ga East Municipal of Greater Accra Region, Ghana, with a total of 30 CHNs. In 2015, $15 \mathrm{CHNs}$ were recruited from two subdistricts (Dome and Taifa) in collaboration with officials from the CHPS programme. These CHNs, who had prior working knowledge of community and health resources, participated in a 2-week onsite introductory VIA/cervicography training modelled after the JHIPEGO course used globally, ${ }^{39}$ plus 3 months smartphone-based mentorship for improving VIA diagnostic and management skills using imaging of the cervix. ${ }^{36}$ We interviewed all CHNs $(n=15)$ who participated in this study. They had 2 years of nurse training and were employed by the Ghana Health Service that participated in this study. Additionally, we used prospective purposive and criteria sampling techniques to recruit patient participants ( $\mathrm{n}=21$, of 169 screened) including those of different ages, those receiving cryotherapy or further treatment $(n=6)$, and from both CHCs. To triangulate data, an expert reviewer and a head nurse were additionally interviewed.
Multiple smartphones were evaluated in a pilot assessment for their quality of imaging, cost and ease of use. The Samsung Duos was the best option available in Ghana at the time of this study with reasonable image resolution, ability to auto and manually focus, LED camera flash and battery life. A protocolised consistent approach to photography and image transfer was established and used throughout. Training in effective photography of the cervix using smartphones and proper lighting and position of patient as well as the camera was provided during the in-person training. During the immediate feedback phase, for each patient, the CHN sent the best image captured to the mentor for feedback; this resulted in an average of 11 images per CHN.

\section{Data collection and procedures}

Data collection included semi-structured individual interviews conducted at the CHCs in either English or a local Ghanaian language with a trained professional interpreter. Nine CHNs participated in an additional focus group. CHNs responded to 10 open-ended questions with probes regarding attitudes, perceptions of and experience with onsite training, cervical cancer screening, smartphone imaging of the cervix, a 3-month mentorship via text messaging, opinions on the feasibility of VIA/cervicography screening at CHCs and at the national level, and overall logistical barriers and further recommendations (for interview tool, please see online supplementary file 1). Topics for the expert reviewer and head nurse included logistical barriers, opportunities and experiences with VIA/cervicography training and mentorship. Patients were posed 10 open-ended questions with directing probes regarding knowledge, attitudes and experience with cervical cancer screening, VIA/cervicography or cryotherapy and imaging of the cervix using smartphones, privacy concerns, stigma and perceptions of community members, and suggestions to improve the screening programme (for interview tool, please see online supplementary file 2). Interviewers (RA and AN) had previously worked with CHNs in the CHPS programme, and had prior knowledge of the programme, working conditions and health system characteristics in the CHCs. At the beginning of each interview, it was made clear that the information from the participants would be deidentified with no direct link between interview data and participants' identifying information so that they could discuss their experience openly. All interviews were audiorecorded. Notes were taken by trained interpreters, translated into English when needed and checked for interpreter accuracy. Informed consent was obtained from all participants. We clarified that participation would not affect participants in any negative way and that they could withdraw answers or from the study at any time and that non-participation would not adversely affect participants.

\section{Data analysis}

Data were analysed by two researchers (RA and HC) using a qualitative descriptive approach and content 
analysis to identify core perceptions, experience and attitudes. We developed preliminary coding, performed critical deliberation about initial codes and reviewed codes for similarities and variations to assure a high level of agreement. Codes fell into distinct but overarching categories. Reviewers independently reviewed and coded all transcripts, then met to review codes, discuss and identify concepts grouped into the specific categories and described and agreed on major important emergent themes. The study was approved by the Noguchi Institute Institutional Review Board in Ghana as well as the IRB of New York University School of Medicine.

\section{Patient and public involvement}

At early stage of this study, formal and informal discussions with patients and non-study staff of CHCs were held to better develop the research question and understand the potential impact of research, and factor in their current experience and priorities to the extent they were relevant and possible.

\section{RESULTS}

Average age for nurses was 28.2 years (SD, 2.9); $100 \%$ female. Average age for the patients was 33.8 years (SD, 7); $69 \%$ were married or had partners; average age at first sexual intercourse was 20.1 years $(\mathrm{SD}, 4.1)$; average lifetime sexual partners was $3.1(\mathrm{SD}, 2)$ and average number of living children 2.1 (SD, 1.6). Analysis of interviews and focus groups with screening providers, patients and health staff revealed distinct but overarching themes.

\section{Perception and experiences of community health nurses}

Quotes supporting findings on the perceptions and experiences of CHNs can be found in table 1. CHNs viewed the onsite training and its format as effective and comfortable. The majority reported that working with a combination of the speculum, mannequins, real patients and flashcards helped prepare them for actual screenings. Although all agreed that learning the pelvic anatomy was interesting, some mentioned that classroom lectures were not contextual, and that they learnt more working with real patients. Majority stated that it was beneficial that the trainer taught them how to use the speculum, take photos and do the gynaecology exam. Largely, nurses did not feel that financial incentives were needed to encourage learning.

Overwhelmingly, they agreed with adequacy of 3 months mentorship and emphasised the importance of having a back-up expert to further interpret the images and mentor them after the initial onsite training. Although some CHNs expressed anxiety about performing the screenings, almost all agreed that having expert support made them and the patients feel more confident during the process and that the expert reviewer's responsiveness and individualised support improved their competency. Most CHNs elaborated that seeing positive VIA cases was very important and was integral in learning, but all agreed that negative cases were easier. CHNs also agreed that almost all patients understood when they were told to wait for the expert to confirm a potentially positive or negative case during mentorship period, as feedback was provided by the mentor either immediately or within 24 hours, depending on availability of the mentor. Another helpful aspect of the 3-month training period was peer-to-peer support from colleagues for diagnostic input.

Overall, the CHNs found smartphones easy to use for cervicography and sending photos to the mentor, and experienced few logistical issues (such as issues with SIM cards, texting or network). Electricity outage was an occasional barrier which was largely addressable. Although some nurses had some initial difficulty with taking quality photos, the expert support helped to improve quality of images over time. CHNs elaborated that describing the process to patients and showing them their cervical pictures made them more assured and comfortable with screening. Almost all CHNs felt that smartphone-based cervicography VIA is very easy to integrate into the screening process. Overall, nurses were proud of their work. They suggested a peer support network to discuss pictures and cases. Nurses overwhelmingly mentioned that they would appreciate having a certificate of training completion to mark their achievement and competency.

CHNs believed that more and more people will come to get screened because it has already received attention, and both information and acceptability seem to be increasing in the community. Nurses believed that they are the most appropriate personnel to administer this service, especially considering the sensitive nature of the procedure, and because of the familiarity and trust they have garnered within communities. CHNs acknowledged that potential screening cost would be a barrier, and that assuring the screening was free of charge was important. They reported a general lack of knowledge of cervical cancer and screening as challenges to attracting patients. CHNs reported that some patients believed that screenings were unnecessary because they were asymptomatic. Other barriers to convincing patients included anticipated pain during the procedure, potential cost and fear of knowing about a positive screening result. CHNs reported that educating patients on cervical cancer and screening, and its importance in preventing cancer helped overcome these barriers. CHNs also relied on their existing trusting relationships with patients to encourage screening. They felt that most patients accepted undressing for the exam, with some patients comparing the nudity to the experience of childbirth. CHNs reported that their patients had minimal privacy concerns with having their cervix photographed, since the picture was anonymous and viewed only by the patient, nurse and the expert reviewer.

Overwhelmingly, nurses did not face any major technical challenges in performing VIA. A minority mentioned that it was difficult at times, but manageable, to find the actual cervix. Logistical challenges included the cost of transportation, both for patients and CHNs; at times, securing a steady supply of equipment and 


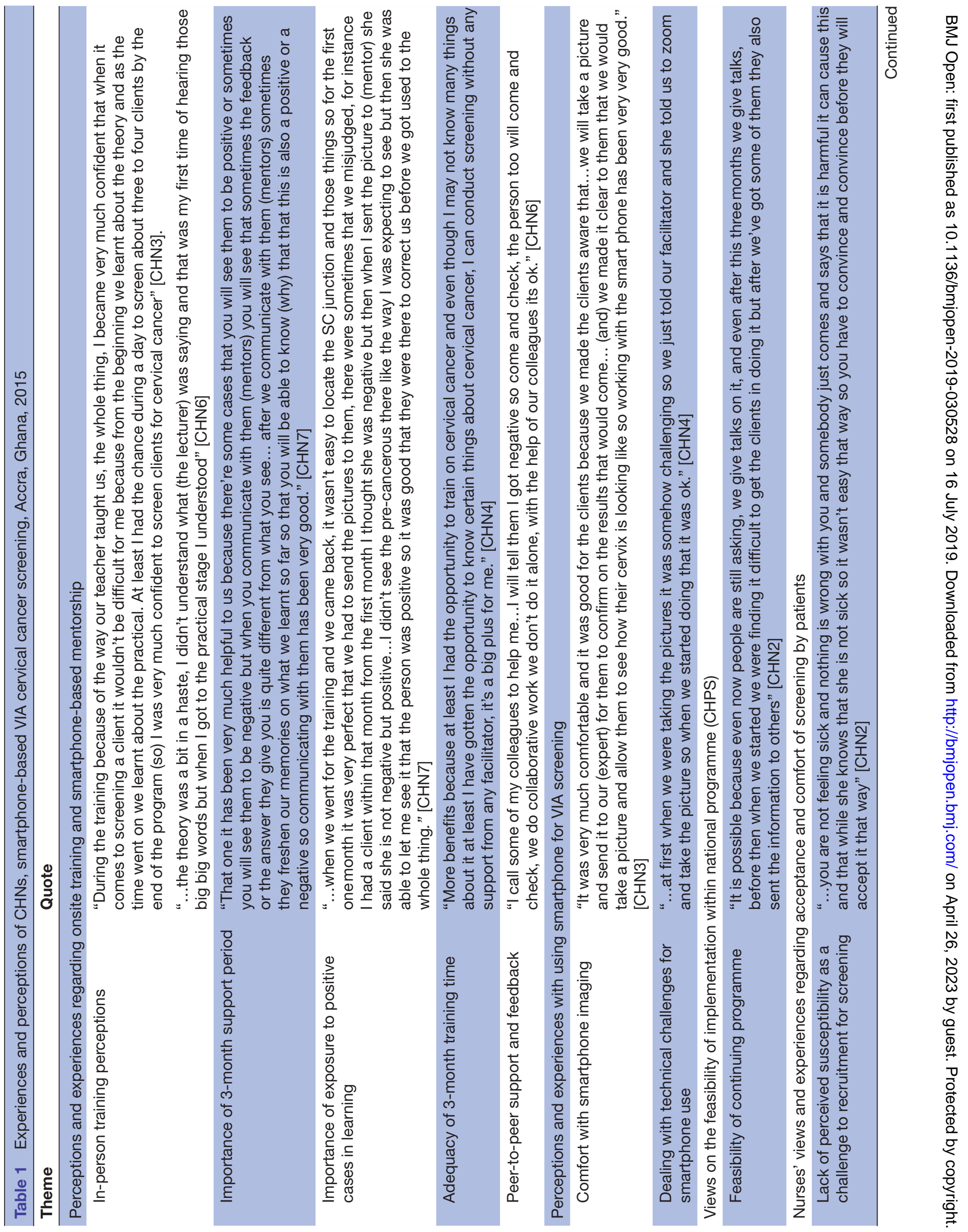




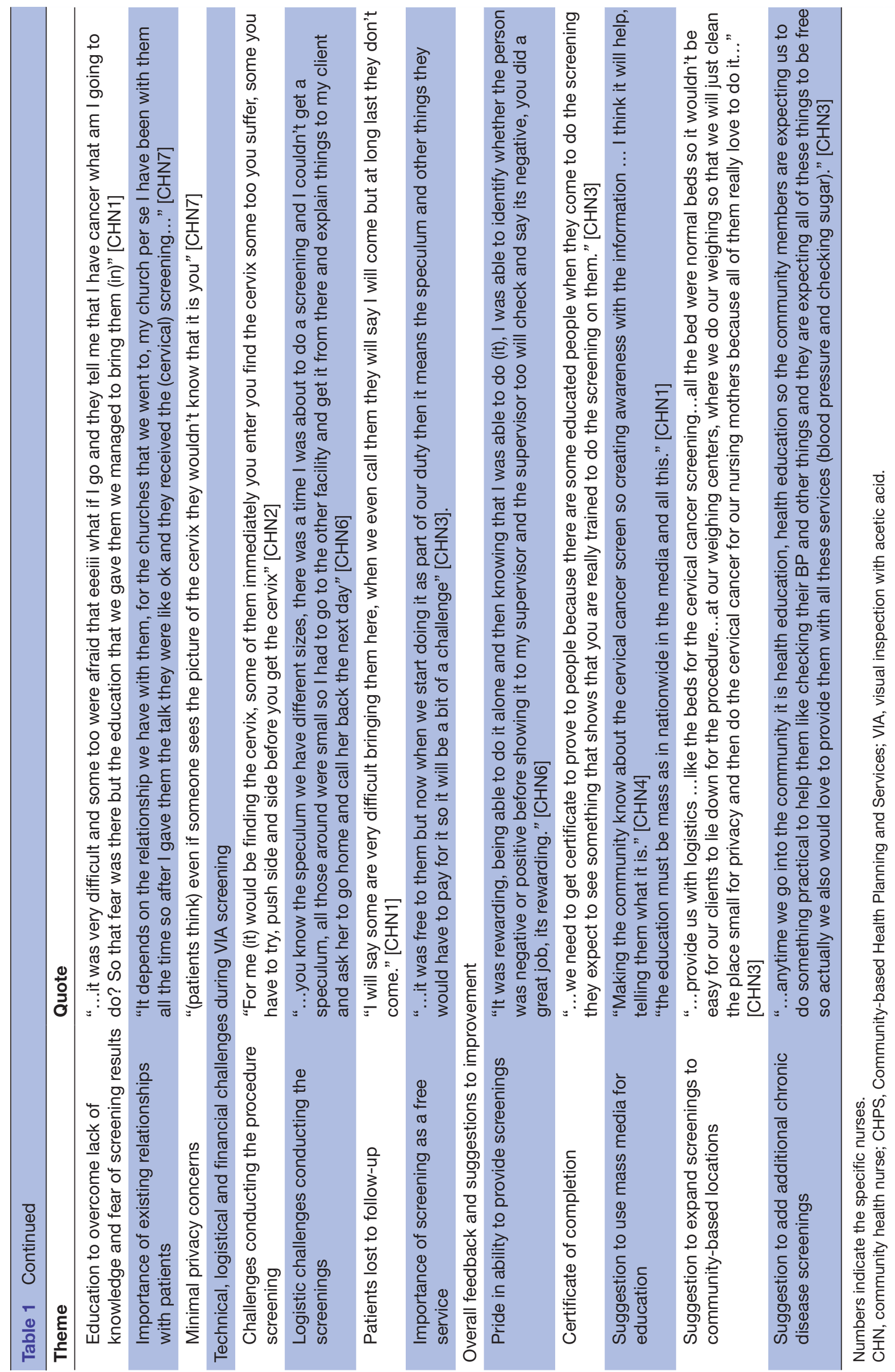

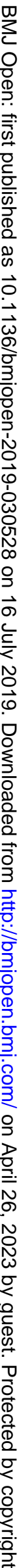


resources; the quality of equipment including exam beds and the relatively small size of the screening area which posed minor challenges to maintaining patient privacy. Commonly used medical exam beds were available in all facilities or mobile units. Lighting was supported by stationery medical exam lamps. Bedsheets, gloves, and other supplies and materials for screening were all part of usual supplies in the clinics/health facilities and used based on Ghana's Ministry of Health recommendations for infection control and OBGYN exam. Smartphones had flashlights and were used for photography in a protocolised fashion developed for this study. None of the CHNs perceived transport time as a barrier for patients; however, the transportation cost was among the most common reasons women missed screening appointments. Almost all CHNs agreed that paying for transportation would improve the screening process. CHNs suggested ways to improve awareness and education by making announcements through radio, TV, newspapers and social media. To improve patient screening participation, they suggested going door-to-door, and reaching out to local churches. They also indicated the need for both nationwide education and community mobilisation. Other improvements of the experience included access to equipment for screening such as better quality, sturdier exam beds; offering screenings at opportune times like when women are at health centres for other reasons, or screening in the community. They suggested expanding screening services to include breast cancer, diabetes and blood sugar levels, hepatitis B and hypertension. Several mentioned a recent reduction in previous services due to a lack of funding, emphasising the importance of keeping these services free to the public.

\section{Perceptions and experiences of the nurse supervisor and expert reviewer}

Both the supervisor and expert reviewer generally agreed with CHNs regarding the appropriateness of the training and its incorporation and adequacy of both theoretical and practical trainings. They added the need to reduce the size of the training groups in order to provide more personalised attention to each training during the initial onsite sessions (groups of 6-7 rather than 15-20). This would also allow the trainer to identify and provide targeted attention to different trainees. The expert reviewer did not cite any logistical challenges with receiving and sending images and mentoring and providing feedback. Actually, the reviewer spent some time helping a few CHNs improve the quality of their photos but she did not find this a significant challenge. She found the process very rewarding and elaborated on the importance of providing 3-month remote mentorship to help advance and further polish CHNs' diagnostic skills as a more practical training that complemented classroom exposure. This in turn improved the reviewer confidence regarding the competence of CHNs and screening quality.

\section{Perception and experiences of patients}

Largely, both patients who were screened negative and those who were screened positive and received cryotherapy shared similar perceptions and experiences. Quotes from patients exemplifying each theme are presented in table 2. None of the patients, including the cryotherapy patients, had ever been screened before, and most had no prior knowledge of cancer or screening. For the few with some prior knowledge, past exposure included TV or radio, or informal information from nurses when visiting health facilities on unrelated occasions. Only a small number of patients understood the concept of screening, prevention and the importance of knowing ones' disease status. Many expressed misconceptions, either their own or beliefs they had heard from others. One patient elaborated on misconceptions, sharing her belief that cervical cancer is caused by inserting things into the vagina. Other misconceptions about screening included worrying about being 'clean' or 'neat' enough to be screened or that nurses would put their hands inside patients. Most patients did not discuss the screening with anyone, and decided to participate in the screening on their own. Only a few asked partners for approval and the majority reported that their husbands or partners supported their decision. They elaborated that some men did not think the screening was relevant to them; however, most, but not all, men in the community support screening. A few said that some men had issues with it but generally if women decided to get screened, then they could find a way without their partner's approval.

Most patients appreciated cervical photography and wanted to see the picture of their own cervix. For many, the photos helped answer their questions and, for some, it reassured them about the procedure. Some women wanted to take their cervical images to show to their partners or keep as a record. Therefore, if and when patients requested to have their cervical digital images and had a smartphone, providers allow them to take the picture or transferred images securely to the patient's smartphone. These images were all deidentified and anonymised. Printing images was not possible or provided. Due to the anonymity, the majority were comfortable with having pictures taken. Only one patient had questions and requested reassurance about potential sharing of her images on the internet. Most others trusted the CHNs and understood and agreed with the process of smartphone-based VIA. Patients were generally appreciative of CHNs' persistence in educating them and addressing misunderstandings and misconceptions through describing the screening process, cancer detection and treatment, and relaying the importance of prevention. Some patients mentioned that the CHNs showing pictures of cancerous lesions and describing risk factors for cervical cancer was often effective in encouraging screening. The majority of patients reported little or no stigma from relatives or community members for getting screened. Although most patients stated that a large number of women in the community support screenings, 


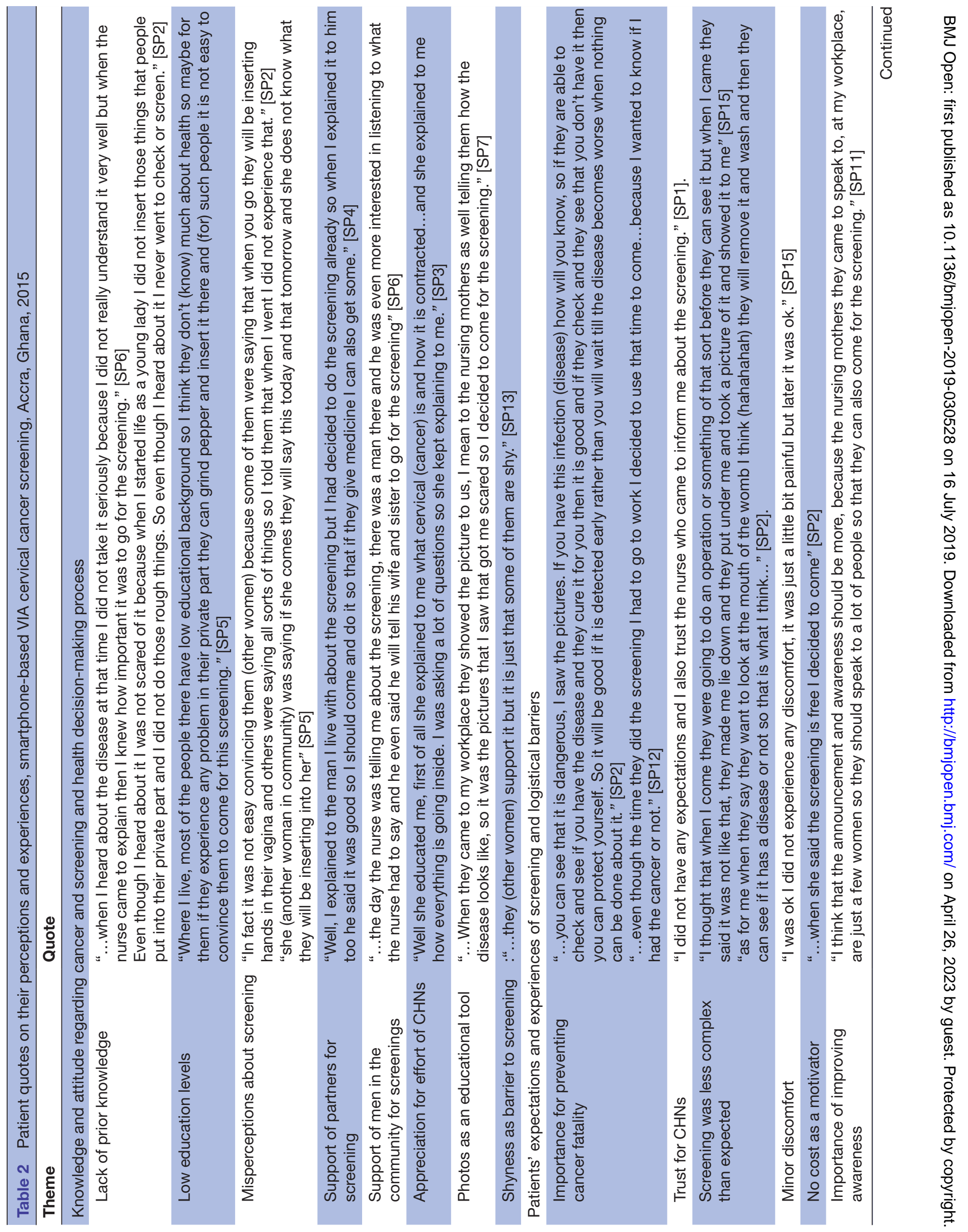


some alluded that barriers included embarrassment or shyness and low health literacy. While some women discussed misconceptions in their community regarding the procedure itself, most clients were optimistic that more men and women would support the screening if they were educated more about the procedure.

Majority of clients had no specific expectations of the screening prior to their procedure, and they largely trusted the CHNs as health professionals. Overall, clients found it easier, shorter, and less painful than expected. Some expected a much more complicated or painful procedure such as that the uterus to be taken out and washed and inspected. Although a few experienced initial discomfort, it was often relatively minor and short-lived. None of the clients had any significant logistical barriers during the screening. A few mentioned issues with transport cost, the majority thought that screening time was reasonable, and appreciated the screening being free. Some clients skipped work to be screened. Majority of clients were happy with the screening, and did not offer any specific recommendations for improvement. Some clients inquired about and suggested providing breast cancer screening. Most of them acknowledged it was important to have more announcements and awareness, in one case suggesting that nurses go house to house in order to educate people. Individual women who received cryotherapy after screening positive through VIA cited positive experiences with the process and did not report any specific privacy issues, or additional pain or discomfort or logistical barriers.

\section{DISCUSSION}

Cervical cancer screening using VIA and smartphone was highly accepted by the clients, none of whom had prior screening, and by CHNs who were performing this screening for the first time. Neither group cited significant barriers to receiving or performing VIA at CHCs, the incorporation of smartphone imaging, the process of mentorship via text messaging, privacy or confidentiality, or other issues with stigma or logistical barriers. Some barriers to screening cited by both clients and CHNs closely resembled those of patients in more developed regions, such as fear, embarrassment or lack of knowledge or awareness. ${ }^{40}$ The training, including initial on-site and subsequent mentorship using smartphone imaging and texting, was very well received. The 3-month mentorship was an important and effective complementary training to further develop and enhance the needed competencies. Both CHNs and their trainer and supervisor regarded the didactic and practical components of the onsite training as interrelated and vital to its effectiveness. CHNs found the training to be empowering and welcomed the opportunity to learn a new skill, and the opportunity to provide additional services to women in their catchment areas. The importance, value and synergistic effect of having continuous diagnostic support in the first 3 months was essential. Smartphone-based 
imaging provided opportunities to see and discuss cases that they did not see during their initial training such as identifying more positive or complicated cases, ${ }^{36-38}$ and provided the opportunity to revisit images and discuss difficult cases with colleagues, all of which facilitated learning. Discussing images with the mentor further reinforced clients' trust, and clients overwhelmingly accepted smartphone-based imaging, had no significant privacy issues and felt more reassured of the procedure.

The possibility to discuss and learn from their own experience, and individualised support and mentorship during this 3-month period improved competency and confidence in managing their clients. Adult learning theory describes the main mechanisms by which adults learn skills when they relate to daily activities and working responsibilities, learning is hands-on and takes place over time, there are peer-to-peer learning opportunities and there are possibilities to ask for and receive targeted feedback related to their cases/questions as opposed to learning in classroom settings. ${ }^{42} 42$ The use of smartphones provided a likely unmatched opportunity to connect new providers to peers and senior providers, and improved access to case-by-case learning. This important mentorship component has been largely missing from widespread standard VIA trainings. ${ }^{43}$ There were additional important and unanticipated benefits of photography including using photos to better communicate with clients about the procedure and gain more trust, further provide targeted health education regarding sexual and reproductive health, and implement some form of quality control. The ease of using smartphones and the lack of serious logistical barriers were important findings on feasibility from the perspective of all stakeholders, and highlight the potential of smartphones and mentorship in improving quality, accuracy and efficacy of VIA. ${ }^{36-38}$ Smartphone imaging could be used to further create a peer-support network such as a virtual journal club to reinforce peer learning and support, which could be used as a measure for quality control. There is also the potential for better effectiveness of VIA strategies through improving adherence and acceptance by clients due to better understanding of the procedure, addressing misconceptions among clients and their partners and possibility for medical record keeping by clients.

The majority of clients had no, or very little, prior knowledge about cervical cancer or screening. The availability of and persistence of CHNs, from their own communities, in providing basic education about the disease, screening and addressing existing misconceptions were essential in ultimately encouraging women to get screened. Although there were important misconceptions, fear of screening or its results, and some stigma, clients were receptive and communicated the overall benefits for themselves and others. Women largely seemed to express autonomy about decisions regarding screening and they were able to manage any potential opposition by their partners without assistance. Negative views and stigma on the part of others (men or women) were not uncommon but were generally not significant barriers. Despite important misinformation regarding the causes of cancer and side effects of the screening, the majority felt that screening strategies were acceptable.

CHNs were generally satisfied with conducting the screening, felt empowered and gained more confidence in their capacities, and saw their efforts as a meaningful component of their jobs. Nurses were keen to be certified and considered that an important factor for career development but also to further gain confidence and trust from clients. All CHNs who participated in this project were women and had experience providing basic maternal and child care and health education to residents of their respective catchment areas. Strong prior relationships with clients and perhaps their gender seemed to foster an ease of the process and better convince clients for screening, and helped address issues of privacy and sensitivity. Equally important compared with knowledge and attitude is trust of providers, which could ultimately encourage clients to make the decision regarding seeking care especially screening for asymptomatic conditions. ${ }^{5}$ CHNs were able to leverage their existing relationships and their own persistency to address a significant lack of knowledge regarding cancer and screening. Women had other barriers and competing priorities such as cost of transport, time constraints and childcare responsibilities. These were often locally managed by women and CHNs working together, and providing some transportation support to clinics for the procedure. Other logistical barriers such as screening supplies were generally managed by existing resources at the centres. Having screening free of charge was an important factor and needs to be factored in for implementation strategies.

Clients and CHNs had some suggestions to improve the experience including providing more and better-quality facilities and mobile clinics at churches or in the community to bypass transport to clinics. However, this needs to be balanced to ensure that quality is not impacted by mobile units. CHNs generally felt positively regarding the feasibility of incorporating VIA screening into existing services as a long-term strategy. Clients requested additional screening programmes such as breast cancer, which was also suggested (with hypertension and diabetes) and deemed feasible by CHNs. Although CHNs felt that they were quite capable and proud of providing the screening to women in their community, they expressed some scepticism regarding funding for maintaining screening programmes. At the national level, priority setting should include a balance between the cost and outcomes and competing programmes. All these findings may have implications for VIA screening strategies and their implementation into regular health system services at the primary care level as opposed to vertical or targeted services at tertiary care centres or flash mass screening programmes. 


\section{Limitations}

Our study was not without limitations. Although the CHPS programme in Ghana was based on previous service delivery models from rural areas, the two pilot study areas were part of an expansion of the programme, in which the model was adapted to peri-urban and urban areas. Thus, findings may not be generalisable to rural areas, particularly given differences by region in access to social and healthcare resources. As this evaluation was a pilot study to evaluate the effectiveness of using mHealth strategies to extend and improve training in VIA to CHNs, additional challenges may be associated with scaling up such an intervention to larger areas. Additionally, there is a possibility of bias towards acceptability of the intervention. However, we included all CHNs in two facilities rather than relying on a self-selected sample, and patients were recruited using criteria sampling. We also included all clients who received cryotherapy to explore their views towards the screening and treatment strategies. Finally, this qualitative study by its nature only generates hypotheses which need to be further tested quantitatively for validity and generalisability. Nevertheless, we were able to observe existing community perceptions through the lens of participating patients and nurses as they were also asked and subsequently elaborated on views and perceptions from their own community different or consistent with that of their own.

\section{CONCLUSIONS}

Our findings indicate overall acceptability and ease of implementing smartphone-based imaging for VIA and training and mentorship of mid-level health personnel for cervical cancer screening. Anticipated barriers, such as concerns for privacy and confidentiality, knowledge and attitude gaps, fears and misconceptions and logistical challenges were largely minimal or manageable. The use of smartphone imaging provided unexpected benefit as an educational tool to increase patients' adherence and improved VIA training for CHNs by providing mentorship and peer-to-peer education opportunities. Overall, and especially for more remote areas, there are important promises for quality improvement and effectiveness of smartphone-based VIA screening and its implementation. Further and complementary context-based implementation research is needed to appropriately test smartphone-based imaging for scale-up efforts and addressing the cervical cancer burden in LMICs.

\footnotetext{
Author affiliations

${ }^{1}$ Global Health, George Washington University Milken Institute of Public Health, Washington, District of Columbia, USA

${ }^{2}$ Medicine, Weill Cornell Medical College, New York, NY, USA

${ }^{3}$ Environmental Science and Technology, Autonomous University of Barcelona Barcelona Catalunya Spain, Barcelona, Spain

${ }^{4}$ Social and Behavioral Sciences, University of Ghana School of Public Health, Accra, Ghana

${ }^{5}$ Population, Family and Reproductive Health, University of Ghana School of Public Health, Accra, Ghana

${ }^{6}$ Dean, University of Ghana School of Public Health, Accra, Ghana
}

${ }^{7}$ Obstetrics and Gynecology, Korle-Bu Teaching Hospital, Accra, Ghana

Acknowledgements The authors extend their appreciation with special thanks to CHCs staff, translators, CHIPS staff and Ghana University School of Public Health for their contribution to the implementation and success of this project. This study received partial funding through Global Health Challenge Fund from New York University Global Institute of Public Health.

Contributors RA and $\mathrm{HC}$ made substantial contributions to this study including conception and design, analysis and interpretation of data, technical and material support and supervision, drafting and critical revision of the manuscript for important intellectual content, and the approval of final version of the manuscript. PA and RAd made substantial contributions to this study including conception and design, technical and material support and supervision, critical revision of the manuscript for important intellectual content, and the approval of final version of the manuscript. AN, EM and AAA made substantial contributions to this study including data collection and technical and material support and supervision and the approval of final version of the manuscript. HB made contribution to the analysis and interpretation of data and the final approval of the manuscript.

Funding This study was partially funded by the New York University Institute of Global Health, Global Health Challenge Fund.

Competing interests None declared.

Patient consent for publication Not required.

Ethics approval This study was approved by the Noguchi Institute Institutional Review Board in Ghana as well as New York University School of Medicine (Protocol \# s14-01466).

Provenance and peer review Not commissioned; externally peer reviewed.

Data sharing statement All data relevant to the study are included in the article or uploaded as supplementary information.

Open access This is an open access article distributed in accordance with the Creative Commons Attribution Non Commercial (CC BY-NC 4.0) license, which permits others to distribute, remix, adapt, build upon this work non-commercially, and license their derivative works on different terms, provided the original work is properly cited, appropriate credit is given, any changes made indicated, and the use is non-commercial. See: http://creativecommons.org/licenses/by-nc/4.0/.

\section{REFERENCES}

1. Farley J, Shin HR, Bray F, et al. Estimated Cancer Incidence, Mortality and Prevalence Worldwide in 2012, France: International Agency for Research on Cancer. Lyon, France: World Health Organization, 2012. Available: http://globocan.iarc.fr/Default.aspx [Accessed 26 Aug 2017].

2. Ginsburg O, Bray F, Coleman MP, et al. The global burden of women's cancers: a grand challenge in global health. Lancet 2017;389:847-60.

3. Sankaranarayanan R, Ferlay J. Worldwide burden of gynaecological cancer: the size of the problem. Best Pract Res Clin Obstet Gynaecol 2006;20:207-25.

4. Forman D, de Martel C, Lacey CJ, et al. Global burden of human papillomavirus and related diseases. Vaccine 2012;30(Suppl 5):F12-23.

5. Ghana Demographic and Health Survey 2008. In: Accra, Ghana: Ghana Statistical Service (GSS), Ghana Health Service (GHS) and ICF Macro, 2009.

6. Hoppenot C, Stampler K, Dunton C. Cervical cancer screening in high- and low-resource countries: implications and new developments. Obstet Gynecol Surv 2012;67:658-67.

7. Ditzian LR, David-West G, Maza M, et al. Cervical cancer screening in low- and middle-income countries. Mt Sinai J Med 2011;78:319-26.

8. Handlogten KS, Molitor RJ, Roeker LE, et al. Cervical cancer screening in Ghana, west Africa: prevalence of abnormal cytology and challenges for expanding screening. Int J Gynecol Pathol 2014;33:197-202.

9. Denny L, Kuhn L, Pollack A, et al. Evaluation of alternative methods of cervical cancer screening for resource-poor settings. Cancer 2000;89:826-33.

10. Miller AB, Sankaranarayanan R, Bosch FX, et al. Can screening for cervical cancer be improved, especially in developing countries? Int J Cancer 2003;107:337-40. 
11. Fokom-Domgue J, Combescure C, Fokom-Defo V, et al. Performance of alternative strategies for primary cervical cancer screening in subSaharan Africa: systematic review and meta-analysis of diagnostic test accuracy studies. BMJ 2015;351:h3084.

12. Nessa A, Hussain MA, Rahman JN, et al. Screening for cervical neoplasia in Bangladesh using visual inspection with acetic acid. Int J Gynaecol Obstet 2010;111:115-8.

13. Firnhaber C, Mayisela N, Mao L, et al. Validation of cervical cancer screening methods in HIV positive women from Johannesburg South Africa. PLoS One 2013;8:e53494.

14. Huchko MJ, Sneden J, Zakaras JM, et al. A randomized trial comparing the diagnostic accuracy of visual inspection with acetic acid to Visual Inspection with Lugol's lodine for cervical cancer screening in HIV-infected women. PLoS One 2015;10:e0118568.

15. Basu P, Mittal S, Banerjee D, et al. Diagnostic accuracy of VIA and HPV detection as primary and sequential screening tests in a cervical cancer screening demonstration project in India. Int $J$ Cancer 2015;137:859-67.

16. Huchko MJ, Sneden J, Sawaya G, et al. Accuracy of visual inspection with acetic acid to detect cervical cancer precursors among HIV-infected women in Kenya. Int J Cancer 2015;136:392-8.

17. Qiao L, Li B, Long M, et al. Accuracy of visual inspection with acetic acid and with Lugol's iodine for cervical cancer screening: Metaanalysis. J Obstet Gynaecol Res 2015;41:1313-25.

18. Mustafa RA, Santesso N, Khatib R, et al. Systematic reviews and meta-analyses of the accuracy of HPV tests, visual inspection with acetic acid, cytology, and colposcopy. Int J Gynaecol Obstet 2016;132:259-65.

19. Moon TD, Silva-Matos C, Cordoso A, et al. Implementation of cervical cancer screening using visual inspection with acetic acid in rural Mozambique: successes and challenges using HIV care and treatment programme investments in Zambézia Province. J Int AIDS Soc 2012;15:17406.

20. Hill AG, Darko R, Seffah J, et al. Health of urban Ghanaian women as identified by the Women's Health Study of Accra. Int J Gynaecol Obstet 2007;99:150-6.

21. Shastri SS, Mittra I, Mishra GA, et al. Effect of VIA screening by primary health workers: randomized controlled study in Mumbai, India. J Natl Cancer Inst 2014;106:dju009.

22. Ministry of Health. National Strategy for Cancer Control in Ghana: 2012-2016. Available: http://www.iccp-portal.org/sites/default/files/ plans/Cancer\%20Plan\%20Ghana\%202012-2016.pdf

23. Blumenthal PD, Gaffikin L, Deganus S, et al. Cervical cancer prevention: safety, acceptability, and feasibility of a single-visit approach in Accra, Ghana. Am J Obstet Gynecol 2007;196:407. e1-407.e9.

24. Ghana Health Service. Community Health Planning and Services (CHPS): The Operational Policy, 2005. Available. http://www. expandnet.net/PDFs/HP25.pdf

25. Nyonator FK, Awoonor-Williams JK, Phillips JF, et al. The Ghana community-based health planning and services initiative for scaling up service delivery innovation. Health Policy Plan 2005;20:25-34.

26. Sanghvi H, Limpaphayom KK, Plotkin M, et al. Cervical cancer screening using visual inspection with acetic acid: operational experiences from Ghana and Thailand. Reprod Health Matters 2008;16:67-77.
27. Odoi-Agyarko H. Profile of Reproductive Health Situation in Ghana. Accra: World Health Organization, Country Office Ghana, 2003.

28. Mwanahamuntu MH, Sahasrabuddhe VV, Kapambwe S, et al. Advancing cervical cancer prevention initiatives in resourceconstrained settings: insights from the Cervical Cancer Prevention Program in Zambia. PLoS Med 2011;8:e1001032.

29. Sherigar B, Dalal A, Durdi G, et al. Cervical cancer screening by visual inspection with acetic acid-interobserver variability between nurse and physician. Asian Pac J Cancer Prev 2010;11:619-22.

30. Bomfim-Hyppólito S, Franco ES, Franco RG, et al. Cervicography as an adjunctive test to visual inspection with acetic acid in cervical cancer detection screening. Int J Gynaecol Obstet 2006;92:58-63.

31. Firnhaber C, Mao L, Levin S, et al. Evaluation of a cervicographybased program to ensure quality of visual inspection of the cervix in HIV-infected women in Johannesburg, South Africa. J Low Genit Tract Dis 2015;19:7-11.

32. Cronjé HS, Cooreman BF, Beyer E, et al. Screening for cervical neoplasia in a developing country utilizing cytology, cervicography and the acetic acid test. Int J Gynaecol Obstet 2001;72:151-7.

33. Cremer M, Jamshidi RM, Muderspach L, et al. Digital camera assessment for detection of cervical intraepithelial neoplasia in rural El Salvador. Int J Gynaecol Obstet 2005;91:42-6.

34. Mwanahamuntu MH, Sahasrabuddhe VV, Blevins $\mathrm{M}$, et al. Utilization of cervical cancer screening services and trends in screening positivity rates in a 'screen-and-treat' program integrated with HIV/ AIDS care in Zambia. PLoS One 2013;8:e74607.

35. Parham GP, Mwanahamuntu MH, Pfaendler KS, et al. eC3--a modern telecommunications matrix for cervical cancer prevention in Zambia. J Low Genit Tract Dis 2010;14:167-73.

36. Asgary R, Adongo PB, Nwameme A, et al. mHealth to Train Community Health Nurses in Visual Inspection With Acetic Acid for Cervical Cancer Screening in Ghana. J Low Genit Tract Dis 2016;20:239-42.

37. Yeates KE, Sleeth J, Hopman W, et al. Evaluation of a SmartphoneBased Training Strategy Among Health Care Workers Screening for Cervical Cancer in Northern Tanzania: The Kilimanjaro Method. BMJ Open 2015;5:e005828.

38. Sharma D, Rohilla L, Bagga R, et al. Feasibility of implementing cervical cancer screening program using smartphone imaging as a training aid for nurses in rural India. Public Health Nurs 2018;35:526-33.

39. Gaffikin L, Blumenthal PD, Emerson M, et al. Safety, acceptability, and feasibility of a single-visit approach to cervical-cancer prevention in rural Thailand: a demonstration project. Lancet 2003;361:814-20.

40. Gesink D, Filsinger B, Mihic A, et al. Cancer screening barriers and facilitators for under and never screened populations: A mixed methods study. Cancer Epidemiol 2016;45:126-34.

41. Taylor DC, Hamdy H. Adult learning theories: implications for learning and teaching in medical education: AMEE Guide No. 83. Med Teach 2013;35:e1561-72.

42. Brookfield SD. Understanding and Facilitating Adult Learning; A comprehensive Analysis of Principles and Effective Practices. Buckingham, UK: Open University Press, 1996.

43. University of Zimbabwe/JHPIEGO Cervical Cancer Project. Visual inspection with acetic acid for cervical-cancer screening: test qualities in a primary-care setting. Lancet 1999;353:869-73. 